\title{
Máster de secundaria: análisis de situación en la universidad española
}

\section{Master's degree in secondary school teaching training: analysis of situation in Spanish Universties}

\author{
Víctor López Ramos ${ }^{1 *}$ \\ vmlopez@unex.es, https://orcid.org/0000-0002-5945-0810 \\ Rocío Yuste Tosina* \\ rocioyuste@unex.es, https://orcid.org/0000-0002-9241-0079 \\ Pello Urkidi Elorrieta** \\ pello.urkidi@ehu.eus,https://orcid.org/0000-0003-1818-8550 \\ Daniel Losada IGLesias** \\ daniel.losada@ehu.eus, https://orcid.org/0000-0003-3842-7694 \\ *Universidad de Extremadura, España \\ **Universidad del País Vasco, España
}

\section{Resumen:}

Este artículo pretende dar respuesta a la preocupación surgida en la Conferencia de Decanos de Educación de las Universidades españolas sobre la implantación y desarrollo del Máster que habilita para la profesión docente en la enseñanza secundaria. Hemos tratado de aportar datos que sirvan como mapa de situación acerca de la realidad de estos estudios y las diferencias en su implantación. El trabajo se ha elaborado a partir de las respuestas obtenidas a un cuestionario que cumplimentaron 35 universidades, de las 72 que imparten el Máster en España. Los resultados muestran una gran heterogeneidad, principalmente, en cuatro aspectos: la oferta de plazas y de especialidades, la estructura del plan de estudios, el perfil profesio-

\begin{abstract}
:
This article aims to respond to the concern raised in the Conference of Deans of Education of Spanish Universities regarding the implementation and development of the Master's Degree in Secondary Education Teacher Training. We aim to provide data that serve as a situation map about the reality of these studies and the differences in their implementation. Data were obtained through a questionnaire completed by 35 universities, of the 72 that teach this master's in Spain. The results show a great heterogeneity, mainly in four aspects: the offer of seats and specialties, the structure of the curriculum, the professional profile of the teaching staff and the development of school trainings and master's theses. The results highlight the need for
\end{abstract}

1 Dirección para correspondencia (correspondence address):

Víctor López Ramos. Departamento de Psicología y Antropología. Universidad de Extremadura. Avd. de la Universidad s/n. 10003 Cáceres (España). 
nal del profesorado que imparte docencia en el mismo y la realización del Practicum y del Trabajo de Fin de Máster. Del estudio, se concluye la necesidad de que el Máster deba gestionarse académicamente por los centros universitarios responsables de la formación inicial del profesorado, así como la exigencia de una clarificación precisa, por parte de las agencias de evaluación, que homogenice los criterios de acreditación para el conjunto de universidades y comunidades autónomas.

\section{Palabras clave:}

Enseñanza secundaria; formación inicial de docentes; planes de estudios; organización curricular; práctica pedagógica. the master's to be managed academically by the university faculties in charge of initial teacher training, as well as the need for precise clarifications, on the part of the evaluation agencies, with a view to homogenizing accreditation processes for all universities.

\section{Keywords:}

Secondary education; initial training of teachers; curricula; curriculum organization; pedagogical practice.

\section{Résumé:}

Cet article prétend répondre à la préoccupation survenue lors de la Conférence des Doyens d'Éducation des Universités espagnoles quant à l'implantation et le développement du Master qui habilite à la profession enseignante du secondaire. Nous avons tenté d'apporter des informations qui servent de tableau de situation au sujet de ces études et des différences dans leur mise en œuvre. Le travail a été élaboré à partir des réponses obtenues à un questionnaire complété par 35 universités des 72 qui dispensent le Master en Espagne. Les résultats montrent une grande hétérogénéité, principalement au niveau de quatre aspects : I'offre de places et de spécialités, la structure du plan d'études, le profil professionnel du professorat qui donne les cours et la réalisation du stage et du travail fin de Master. La conclusion de cette étude porte sur la nécessité que le Master soit académiquement géré par les centres universitaires responsables de la formation initiale du professorat, ainsi que l'exigence d'une clarification précise de la part des agences d'évaluation, qui homogénéisent les critères d'accréditation pour l'ensemble des universités et régions autonomes.

\section{Mots clés :}

Enseignement secondaire, formation initiale des professeurs, plan d'études, organisation des programmes, pratique pédagogique.

Fecha de recepción: 1-11-2018

Fecha de aceptación: 12-10-2019

\section{Introducción}

La Conferencia de Decanos de Educación es una entidad que agrupa a todos los centros universitarios españoles, tanto públicos como privados, que imparten títulos de educación: los Grados en Educación Primaria, Educación Infantil, Educación Social y Pedagogía y el Máster Universitario en Formación del Profesorado de Educación Secundaria Obligatoria, 
Bachillerato, Formación Profesional y Enseñanzas de Idiomas (en adelante MUFPES).

Una de sus funciones es analizar y debatir sobre los aspectos que atañen al desarrollo de los títulos que imparten en el ámbito de la Educación. Por ello, en la Asamblea General de la Conferencia, celebrada en la Facultad de Educación de la Universidad de Cantabria en octubre de 2016, se acordó realizar un estudio de la situación del MUFPES, que permitiese conocer la realidad del mismo, generar un debate sobre su implantación en el conjunto de las universidades españolas y, sobre todo, hacer propuestas de mejora que se fundamentaran en datos y no en opiniones u ocurrencias. Este trabajo se encargó a la Facultad de Formación del Profesorado de la Universidad de Extremadura.

Este estudio ha tratado de desgranar los principales elementos y características que configuran el MUFPES, el desarrollo seguido en su implantación y las consideraciones que las diferentes agencias de calidad han realizado a la hora de la verificación de la acreditación del título. Igualmente, se ofrece un análisis comparativo entre las distintas universidades.

Es importante tener en cuenta datos que ofrece el Informe Anual que publica el Sistema Integrado de Información universitaria (2015):

- un crecimiento de estudiantes en el campo de las Ciencias Sociales y Jurídicas, debido principalmente a dos másteres: el MUFPES y el de la abogacía. Ambos suponen el 30\% del total de estudiantes de posgrado de esta rama, mientras que en el resto de las ramas se percibe una clara disminución del número de matriculados.

- la proliferación de universidades privadas en los últimos años en España, muchas de carácter virtual, que han incorporado este título de forma generalizada a su oferta.

Es evidente que, en cualquier sistema educativo, la formación del profesorado debe tener un carácter sistémico, que englobe el acceso a la profesión docente, las necesidades formativas del profesorado novel y la formación continuada del profesional en ejercicio. Pero, aunque no debe olvidarse esta visión holística, la formación inicial es una pieza fundamental en el engranaje de lo que es y debe ser un docente. Por ello, cobra especial significación la idea de que, para la etapa de secundaria, debe ir más allá de la capacitación en aspectos psico-socioeducativos y en didácticas específicas; de hecho, un estudio de Martínez y Villardón- 
Gallego (2015) indica que son los propios estudiantes del título los que identifican dos funciones esenciales en la profesión.

Pero, siguiendo a Tiana (2013), es importante reseñar que en España los modelos adoptados para la formación de maestros y profesores de secundaria difieren notablemente. Así, mientras en el primer caso, se utiliza un modelo integrado que contempla los conocimientos científicos, didácticos, psicopedagógicos..., en el segundo, nos encontramos con un modelo sucesivo, en el que prima la formación disciplinar (240 créditos), que se completa con un curso de posgrado (MUFPES) que trata de atender las necesidades formativas de tipo didáctico y psicopedagógico, con sólo 60 créditos. Según López-Gay, Pro y Rivero (2018) este planteamiento -tanto la secuenciación como la descompensación en la duración- refuerza la idea de que "el que sabe, sabe enseñar". A pesar de las críticas, sigue siendo, como indica Tiana (2013), el modelo con mayor tradición en España y, en principio, parece que no está prevista otra alternativa.

Por otro lado, El Libro Blanco de la Profesión Docente y su Entorno Escolar (Marina, Pellicer y Manso, 2015) ofrece una exhaustiva radiografía -desde nuestra perspectiva, a veces excesivamente negativa- sobre nuestro sistema educativo. Los autores defienden que existe un escaso interés de las universidades por cuidar la calidad y el desarrollo del título, a pesar de que, en muchos casos, los estudiantes de este máster suponen casi el $40 \%$ del total de alumnos matriculados en los posgrados de dichas instituciones. Al respecto, hemos de señalar que no se puede hablar de un "sólo MUFPES", ya que existen desarrollos de programas de formación muy diferentes según la universidad de que se trate, sin que la calidad sea el eje directriz, sino más bien factores académicos (cargas docentes de los Departamentos) o económicos (Marina, Pellicer y Manso, 2015).

Aún con todo, la investigación de Martínez y Villardón-Gallego (2015) destaca que los alumnos del título objeto de nuestro estudio, han cambiado su concepto de la profesión docente, haciéndoles conscientes de las funciones docentes, de la importancia social que tiene -o que debería tener- el profesorado, de los problemas que hay que abordar en este ámbito profesional e igualmente del esfuerzo y compromiso que esta exige. 


\section{Marco teórico: Aspectos generales del título}

\section{Marco legal}

En el Consejo Europeo de Lisboa se establecieron las bases para una adecuada actualización de la enseñanza secundaria en el marco de la Unión Europea (Consejo Unión Europea, 2000), buscando introducir propuestas innovadoras que permitieran una mayor calidad, tanto en los procesos como en la organización y, por supuesto, en los resultados (Blázquez, 2001; Guttman, 2003; Hargreaves, 2003). Para ello, el denominado proceso de Copenhague (Comisión Europea, 2002) pretendía una renovada y fortalecida cooperación en materia de educación entre los estados de la UE.

En España, este intento se materializa en una nueva normativa reguladora general, la Ley Orgánica de Educación (LOE, 2006), en la que se hace referencia a la necesidad de una formación específica para el profesorado de esta etapa educativa.

Por lo tanto, de acuerdo con los planteamientos del Espacio Europeo de Enseñanza Superior (EEES) y las propuestas derivadas de la LOE, se asume la necesidad de una formación psicopedagógica, que complete la disciplinar adquirida en los grados correspondientes. Como respuesta a esta necesidad, aparece un máster para la formación inicial del profesorado que puede cursarse en un año académico completo. En principio, parece que se intenta conferir una mayor importancia a la formación profesional de los futuros profesores; de hecho, tiene una mayor presencia el Prácticum, en comparación con la que tenía en el antiguo Curso de Actitud Pedagógica (CAP): un 40\% en el máster frente al 10\% del CAP (Valle y Manso, 2011).

En cualquier caso, responde a un modelo consecutivo, toda vez que la formación disciplinar antecede temporalmente a la docente. Esto rompe la tendencia en los países de la UE, en los que predomina un modelo concurrente (Eurydice, 2009), en el que convergen la formación disciplinar, didáctica y pedagógica de una manera simultánea (Murillo, 2006; Domingo 2008).

En definitiva, el MUFPES es un título de posgrado perteneciente a la rama de Ciencias Sociales y Jurídicas. Está regulado por la Orden ECI 3858/2007, de 27 de diciembre, que establece los requisitos para la verificación de los títulos universitarios oficiales que habiliten para el ejerci- 
cio de las profesiones de Profesor de Educación Secundaria Obligatoria y Bachillerato, Formación Profesional y Enseñanzas de Idiomas. Además, y su desarrollo se regula a través de dos Reales Decretos:

- Real Decreto 1834/2008, de 8 de noviembre, que define las condiciones de formación para el ejercicio de la docencia en la educación secundaria obligatoria, el bachillerato, la formación profesional y las enseñanzas de régimen especial y en el que se establecen las especialidades de los cuerpos docentes de enseñanza secundaria.

- Real Decreto 665/2015 por el que se desarrollan determinadas disposiciones relativas al ejercicio de la docencia en la Educación Secundaria Obligatoria, el Bachillerato, la Formación Profesional y las enseñanzas de régimen especial, a la formación inicial del profesorado y a las especialidades de los cuerpos docentes de Enseñanza Secundaria.

\section{Estructura de los estudios}

La Orden ECI 3858/2007, de 27 de diciembre, establece, en su Apartado 5 , que los títulos a que se refiere la misma serán enseñanzas universitarias oficiales de Máster con una duración de 60 créditos europeos (ECTS), de acuerdo con el artículo 5 del Real Decreto 1393/2007, de 29 de octubre. Así, estas enseñanzas se estructuran teniendo en cuenta las materias y ámbitos docentes de la actual enseñanza secundaria.

La Orden también establece que, con carácter general, las enseñanzas han de ser presenciales, al menos, en el $80 \%$ de los créditos totales del título. Determina igualmente que todas las Universidades que oferten el Máster deben garantizar que el Practicum tenga carácter presencial y que debe realizarse en colaboración con las instituciones educativas a través de convenios entre las Universidades y las Administraciones Educativas.

En cuanto al plan de estudios, se indican las competencias que deben adquirirse, las cuales se distribuyen atendiendo a la estructura de módulos que recoge la Tabla 1. 
Tabla 1

Módulos y Créditos

\begin{tabular}{|c|c|}
\hline Módulo & $\begin{array}{c}\mathrm{N}^{\mathrm{o}} \text { de créditos } \\
\text { europeos }\end{array}$ \\
\hline $\begin{array}{l}\text { Genérico } \\
\text { Aprendizaje y desarrollo de la personalidad } \\
\text { Procesos y contextos educativos } \\
\text { Sociedad, familia y educación }\end{array}$ & 12 \\
\hline $\begin{array}{l}\text { Específico } \\
\text { Complementos para la formación disciplinar } \\
\text { Aprendizaje y enseñanza de las materias correspondientes } \\
\text { Innovación docente e iniciación a la investigación educativa }\end{array}$ & 24 \\
\hline $\begin{array}{l}\text { Practicum } \\
\text { Practicum en la especialización, incluyendo el Trabajo de Fin } \\
\text { de Máster }\end{array}$ & 16 \\
\hline
\end{tabular}

Por otro lado, la citada Orden establece también que, para el ingreso en el Máster, es necesario estar en posesión de alguna de las titulaciones universitarias que se correspondan con la especialización elegida y que, en caso contrario, deberá acreditarse el dominio de las competencias referentes a la especialización que se desee cursar, a través de la realización de una prueba diseñada por las Universidades.

Igualmente, debe acreditarse el dominio de una lengua extranjera -a nivel B1 del Marco Común Europeo de Referencia para las Lenguas- de acuerdo con la Recomendación No R (98)6 del Comité de Ministros de Estados Miembros de 17 de octubre de 2000.

\section{Marco empírico}

En este estudio nos planteamos el siguiente objetivo general:

- Analizar la situación actual del Máster Universitario de Formación del Profesorado de Educación Secundaria Obligatoria, Bachillerato, Formación Profesional y Enseñanza de Idiomas, en las diferentes universidades del estado.

De manera específica se plantean los siguientes objetivos:

- Describir la oferta de plazas y especialidades.

- Describir la situación actual de la estructura de los estudios. 
- Describir la situación actual del profesorado.

- Describir la situación actual de las prácticas y del Trabajo de Fin de Máster.

\section{Muestra}

Para la realización de este estudio se han tenido en cuenta los datos del Ministerio de Educación, Cultura y Deporte (MECD), concretamente el Informe Anual que publica el Sistema Integrado de Información universitaria (2015). Aunque es posible que existan sensibles diferencias con los datos al día de hoy, no creemos que supongan un cambio espectacular respecto a los mismos.

En el informe mencionado se indica que, en aquel momento, existían 81 universidades con docencia regular (con 243 campus presenciales y 113 sedes no presenciales o especiales) y 2 universidades sin docencia regular. De las 83 universidades, 50 eran de titularidad pública (48 de carácter presencial) y 33 privada (27 de carácter presencial). En total había 1042 centros, de los que 26 correspondían a universidades no presenciales.

En la Tabla 2 se refleja el número de estudiantes estimados para los estudios de máster en la universidad española, de acuerdo con el citado Informe Anual del MECD:

Tabla 2

Estudiantes de máster en las universidades españolas

\begin{tabular}{lll}
\hline & $\begin{array}{l}\text { Alumnado } \\
\text { matriculado en } \\
\text { Máster }\end{array}$ & $\begin{array}{l}\text { Alumnado matriculado } \\
\text { en Máster. Rama Ciencias } \\
\text { Sociales y Jurídicas }\end{array}$ \\
\hline Total & 120.055 estudiantes & 67.337 estudiantes \\
Universidades Públicas & $\begin{array}{l}\text { 86.753 estudiantes } \\
\text { Universidades privadas }\end{array}$ & $\begin{array}{l}33.302 \text { estudiantes } \\
\text { 24.804 estudiantes }\end{array}$ \\
\hline
\end{tabular}

Fuente: Adaptado de SIIU (2015)

Según los datos del Registro de Universidades, Centros y Títulos (RUCT), en el curso 2017-2018, el MUFPES se imparte en 69 de las 81 universidades españolas, existiendo títulos conjuntos impartidos en Universidades de Cataluña y de Canarias.

En la Tabla 3 se recoge la presencialidad del título en las universidades públicas y privadas. 
Tabla 3

Carácter de las universidades que imparten el máster

\begin{tabular}{ccc}
\hline & Presenciales & No presenciales \\
\hline 44 públicas & 43 & 1 \\
25 privadas & 20 & 5 \\
69 totales & 63 & 6 \\
\hline
\end{tabular}

Fuente: Elaboración Propia

Los datos que hemos analizado corresponden a los 35 centros que han respondido nuestra encuesta, que recogemos en la Tabla 4 . A la vista del porcentaje que supone respecto a la población de referencia, podemos considerarla una muestra representativa.

Tabla 4

Carácter de las universidades que han respondido al instrumento

\begin{tabular}{lll}
\hline & Presenciales & No presenciales \\
\hline 27 públicas & 26 & 1 \\
8 privadas & 5 & 3 \\
35 totales & 31 & 4 \\
\hline
\end{tabular}

Fuente: Elaboración Propia

En la Tabla 5 se identifican la universidad, el curso de implantación del MUFPES, el carácter y la modalidad de la muestra que ha respondido al instrumento.

Tabla 5

Datos identificativos

\begin{tabular}{cccc}
\hline UNIVERSIDAD & CURSO & \multirow{2}{*}{ CARÁCTER } & MODALIDAD \\
\hline A Coruña & $2009-10$ & Pública & Presencial \\
Autónoma de Barcelona & $2009-10$ & Pública & Presencial \\
Autónoma de Madrid & $2009-10$ & Pública & Presencial \\
Barcelona & $2009-10$ & Pública & Presencial \\
Burgos & $2009-10$ & Pública & Presencial \\
Cádiz & $2009-10$ & Pública & Presencial \\
Cantabria & $2009-10$ & Pública & Presencial
\end{tabular}




\begin{tabular}{|c|c|c|c|}
\hline UNIVERSIDAD & $\begin{array}{c}\text { CURSO } \\
\text { IMPLANTACIÓN }\end{array}$ & CARÁCTER & MODALIDAD \\
\hline Complutense de Madrid & $2009-10$ & Pública & Presencial \\
\hline Córdoba & 2009-10 & Pública & Presencial \\
\hline Deusto & $2009-10$ & Privada & Presencial \\
\hline Europea & $2009-10$ & Privada & Semipresencial \\
\hline Extremadura & $2009-10$ & Pública & Presencial \\
\hline Francisco de Vitoria & $2010-11$ & Privada & Presencial \\
\hline Girona & $2009-10$ & Pública & Presencial \\
\hline Granada & $2009-10$ & Pública & Presencial \\
\hline Internacional de la Rioja & 2016-17 & Privada & Online \\
\hline Islas Baleares & $2009-10$ & Pública & Presencial \\
\hline La Laguna & $2009-10$ & Pública & Presencial \\
\hline Las Palmas de Gran Canaria & $2009-10$ & Pública & Presencial \\
\hline León & $2009-10$ & Pública & Presencial \\
\hline Málaga & $2009-10$ & Pública & Presencial \\
\hline Murcia & $2009-10$ & Pública & Presencial \\
\hline Navarra & $2009-10$ & Privada & Semipresencial \\
\hline Oviedo & $2009-10$ & Pública & Presencial \\
\hline País Vasco, UPV/EHU & $2009-10$ & Pública & Presencial \\
\hline Pontificia Comillas & $2009-10$ & Privada & Presencial \\
\hline Pontificia de Salamanca & $2009-10$ & Privada & Presencial \\
\hline Pública de Navarra (UPNA) & 2008-09 & Pública & Presencial \\
\hline Ramón Llull & $2009-10$ & Privada & Presencial \\
\hline Salamanca & $2009-10$ & Pública & Presencial \\
\hline Santiago de Compostela & $2009-10$ & Pública & Presencial \\
\hline UNED & 2009-10 & Pública & Virtual \\
\hline Valencia & $2009-10$ & Pública & Presencial \\
\hline Valladolid & 2010-11 & Pública & Presencial \\
\hline Zaragoza & 2009-10 & Pública & Presencial \\
\hline
\end{tabular}

Fuente: Elaboración propia

\section{Instrumento}

Para este estudio se ha elaborado un cuestionario ad hoc que se puede consultar en el siguiente enlace: http://goo.gl/forms/TUFyBu$79 c Y V s w 9 u r 1$. El instrumento fue validado por un grupo de expertos de 
la Comisión Permanente de la Conferencia de Decanos de Educación. El resultado final de la validación cuenta con 7 dimensiones y 53 ítems con la estructura recogida en la Tabla 6.

Tabla 6

Dimensiones e ítems del cuestionario

\begin{tabular}{cc}
\hline Dimensión & Items \\
\hline I. Aspectos sociodemográficos & 10 \\
II. Estructura de los estudios y funcionamiento & 4 \\
III. Profesorado & 6 \\
IV. Desarrollo de las prácticas y trabajo fin de máster & 16 \\
V. Indicadores de calidad & 12 \\
VI. Experiencias innovadoras y buenas prácticas & 1 \\
VII. Renovación de la acreditación & 4 \\
\hline
\end{tabular}

Fuente: Elaboración propia

Los ítems no tienen escalas y se responden mediante preguntas abiertas. Para el presente artículo se han analizado los datos de las dimensiones I, II, III y IV.

\section{Procedimiento}

En la investigación diferenciamos cuatro fases (Rodríguez, Gil y García, 1996): Fase exploratoria; Trabajo de campo; Fase analítica; y Fase informativa.

La primera fase es de índole reflexiva y en ella se diseñó y validó el instrumento de recogida de información ad hoc, al que alude el apartado anterior.

La segunda consistió en el trabajo de campo para identificar a la población y seleccionar la muestra objeto de estudio; posteriormente se procedió al envío, vía correo electrónico, del cuestionario a todas las universidades que imparten el MUFPES.

La tercera comienza con la recogida de información. Para garantizar la fiabilidad se conectó con las fuentes para depurarla. Depurados los datos, se analizaron.

Por último, en la cuarta (https://sites.google.com/site/monografico edu- 
cacionsecundaria/) se exponen los datos, se discuten los resultados, se establecen conclusiones y se realizan propuestas de mejora para el futuro.

\section{Resultados y Discusión}

Los resultados están organizados en los diferentes objetivos específicos planteados. Por otro lado, se contrastan con los valores del único informe oficial que se ha realizado sobre el máster: "Sobre la implantación del Máster Universitario de Formación del Profesorado de Educación Secundaria Obligatoria, Bachillerato, Formación Profesional y Enseñanza de Idiomas. Análisis de situación y propuestas de mejora", elaborado por la Agencia Nacional de Evaluación de la Calidad y Acreditación (ANECA) en 2015.

\section{Objetivo específico 1. Describir la oferta de plazas y especialidades en el MUFPES}

En primer lugar, destacamos el número de especialidades que actualmente se están impartiendo en las diferentes universidades; para ello, utilizamos la Figura 1.

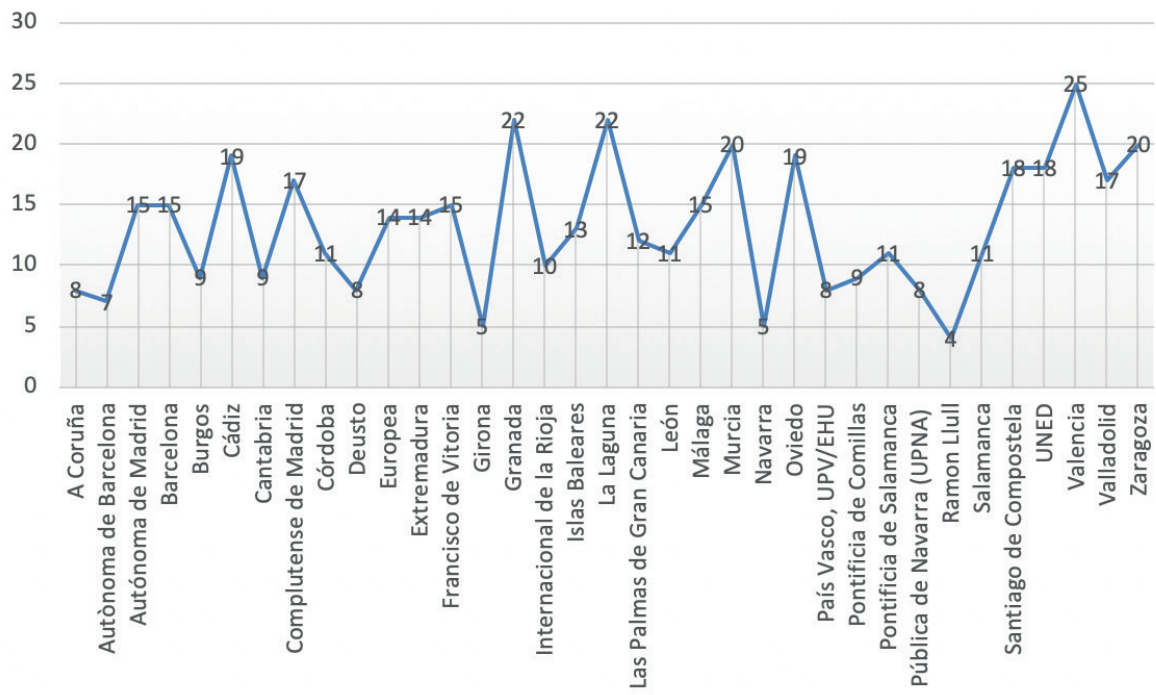

Figura 1. Número de especialidades de cada universidad. Fuente: Elaboración propia. 
Puede observarse que la Universidad que ofrece más especialidades es Valencia (25) y la que menos la de Ramón Llull (4). La media es 13, pero existe una gran heterogeneidad: la mencionada Valencia, Granada, La Laguna, Murcia y Zaragoza (con $\geq 20$ ) y Ramón Llull, Girona, Navarra y Autónoma de Barcelona (con $\leq 7)$.

Este dato llevó a conocer detalladamente las especialidades impartidas, recogidas en la Figura 2.

Como puede observarse, las especialidades más impartidas son Lengua Castellana y Literatura, Inglés, Tecnología y Geografía e Historia. Por otro lado, las menos impartidas serían Construcciones Civiles y Edificación, Griego y Latín.

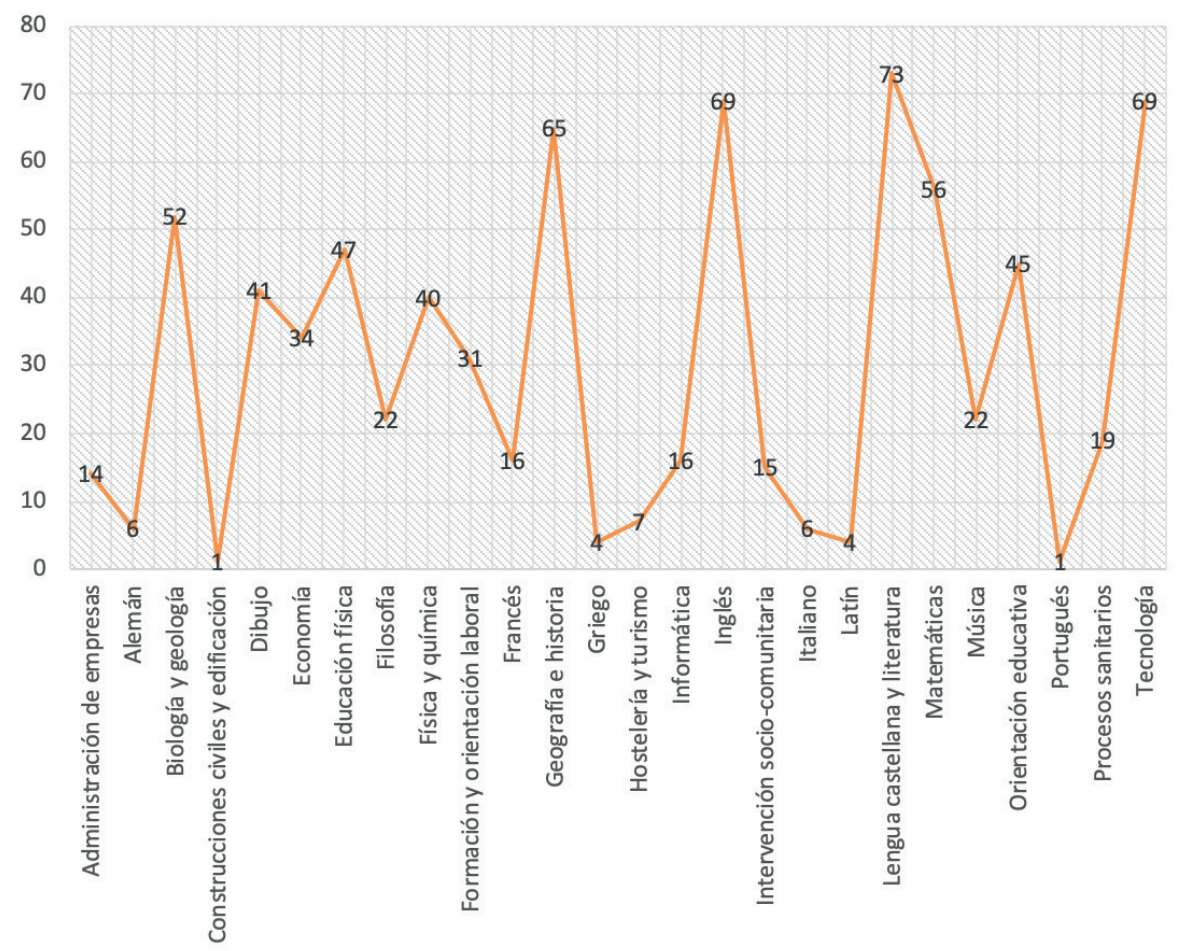

Figura 2. Número de especialidades en el total de la universidad. Fuente: Elaboración propia.

No obstante, existen algunas especialidades, todas en el ámbito de la Formación Profesional, que no se han impartido desde la implantación del Máster en ninguna universidad. Este dato está acorde con el informe de ANECA (2015, p. 19), que asegura: 
Al multiplicarse las ofertas de estudios de Máster las universidades españolas, se han encontrado con dos problemas: la escasa demanda de alumnado para algunas especialidades, y las dificultades para disponer de los recursos humanos y materiales para ofertarlas todas o un número elevado de ellas.

En la actualidad, se puede destacar la propuesta interuniversitaria conjunta de las Universidades de Barcelona, Autónoma de Barcelona, Politécnica de Catalunya, Pompeu Fabra y Oberta de Catalunya y de la Universidad de La Laguna y la Universidad de Las Palmas de Gran Canaria.

En relación a las plazas ofertadas y las ocupadas, los resultados se recogen en la Tabla 7.

Tabla 7.

Oferta de plazas y plazas matriculadas

\begin{tabular}{lccc}
\hline UNIVERSIDADES & NÚMERO & $\begin{array}{c}\text { PLAZAS } \\
\text { OFERTADAS }\end{array}$ & $\begin{array}{c}\text { PLAZAS } \\
\text { MATRICULADAS } \\
\text { (ESTIMADAS) }\end{array}$ \\
\hline $\begin{array}{l}\text { Universidades que } \\
\text { imparten el máster }\end{array}$ & 69 & 20.771 & 17.549 \\
\hline $\begin{array}{l}\text { Universidades que } \\
\text { responden el cuestionario }\end{array}$ & 35 & 11.773 & 10.728 \\
\hline
\end{tabular}

Fuente: Elaboración propia

En las diferentes universidades las plazas ofertadas y matriculadas quedarían repartidas de la manera que recoge el Anexo 1.

Es importante destacar las 1.800 plazas que oferta la Universidad Internacional de la Rioja (se matriculan 1500), las 1.000 plazas de la Universidad de Valencia (se matriculan 923) o las 800 de la Universidad de Granada (se matriculan 800), frente a las 60 de la Pontificia de Comillas o las 40 de la Universidad de Navarra.

Estos datos contradicen el informe de ANECA (2015) que señala que, a la hora de ofertar plazas en estudios profesionalizadores, la ratio no debe ser superior a 25 ó 30 alumnos. Además, según esta agencia, al ser un requisito para el acceso a los Cuerpos Docentes no universitarios, la oferta en cada CC.AA. debe basarse en la previsible demanda de puestos de trabajo a medio plazo en su ámbito territorial (incluyendo la educación pública, la concertada y la privada. 


\section{Objetivo específico 2. Describir la estructura de los estudios del MUFPES}

En la Tabla 8 se refleja la estructura del plan de estudios en las diferentes Universidades; distinguimos los tres módulos.

Tabla 8

Estructura de las enseñanzas

\begin{tabular}{|c|c|c|c|c|c|c|}
\hline \multirow[b]{2}{*}{ UNIVERSIDAD } & \multicolumn{2}{|c|}{ Módulo genérico } & \multicolumn{2}{|c|}{ Módulo específico } & \multicolumn{2}{|c|}{ Practicum } \\
\hline & $\begin{array}{c}\mathrm{N}^{\circ} \text { de } \\
\text { materias }\end{array}$ & $\begin{array}{c}\text { Créditos } \\
\text { en el } \\
\text { módulo }\end{array}$ & $\begin{array}{l}\mathrm{N}^{\circ} \text { de } \\
\text { materias }\end{array}$ & $\begin{array}{c}\text { Créditos } \\
\text { en el } \\
\text { módulo }\end{array}$ & $\begin{array}{c}\mathrm{N}^{\circ} \text { de } \\
\text { materias }\end{array}$ & $\begin{array}{l}\text { Créditos } \\
\text { en el } \\
\text { módulo }\end{array}$ \\
\hline A Coruña & 7 & 20 & 6 & 22 & 2 & 18 \\
\hline $\begin{array}{c}\text { Autónoma de } \\
\text { Barcelona }\end{array}$ & 3 & 15 & 1 & 27 & 2 & 18 \\
\hline $\begin{array}{c}\text { Autónoma de } \\
\text { Madrid }\end{array}$ & 3 & 14 & 3 & 26 & 2 & 20 \\
\hline Barcelona & 3 & 15 & 3 & 25 & 1 & 20 \\
\hline Burgos & 3 & 12 & 6 & 28 & 2 & 20 \\
\hline Cádiz & 3 & 12 & 3 & 24 & 1 & 14 \\
\hline Cantabria & 4 & 15 & 4 & 24 & 2 & 21 \\
\hline $\begin{array}{l}\text { Complutense de } \\
\text { Madrid }\end{array}$ & 3 & 12 & 2 & 30 & 2 & 18 \\
\hline Córdoba & 3 & 12 & 3 & 24 & 2 & 16 \\
\hline Deusto & 3 & 16 & 4 & 26 & 2 & 18 \\
\hline Europea & 3 & 12 & 3 & 24 & 2 & 24 \\
\hline Extremadura & 2 & 12 & 4 & 30 & 2 & 18 \\
\hline $\begin{array}{c}\text { Francisco de } \\
\text { Vitoria }\end{array}$ & 3 & 14 & 3 & 28 & 3 & 18 \\
\hline Girona & 4 & 15 & 8 & 25 & 2 & 20 \\
\hline Granada & 3 & 12 & 3 & 24 & 2 & 16 \\
\hline $\begin{array}{l}\text { Internacional de } \\
\text { la Rioja }\end{array}$ & 3 & 15 & 3 & 24 & 2 & 21 \\
\hline Islas Baleares & 5 & 15 & 7 & 25 & 2 & 20 \\
\hline La Laguna & 3 & 15 & 3 & 24 & 3 & 21 \\
\hline $\begin{array}{l}\text { Las Palmas de } \\
\text { Gran Canaria }\end{array}$ & 3 & 15 & 3 & 24 & 3 & 21 \\
\hline León & 3,5 & 16 & 3,5 & 28 & 2 & 16 \\
\hline Málaga & 3 & 12 & 3 & 24 & 2 & 16 \\
\hline Murcia & 5 & 15 & 6 & 27 & 3 & 18 \\
\hline
\end{tabular}




\begin{tabular}{|c|c|c|c|c|c|c|}
\hline \multirow[b]{2}{*}{ UNIVERSIDAD } & \multicolumn{2}{|c|}{ Módulo genérico } & \multicolumn{2}{|c|}{ Módulo específico } & \multicolumn{2}{|c|}{ Practicum } \\
\hline & $\begin{array}{c}\mathrm{N}^{\mathrm{o}} \mathrm{de} \\
\text { materias }\end{array}$ & $\begin{array}{c}\text { Créditos } \\
\text { en el } \\
\text { módulo }\end{array}$ & $\begin{array}{l}\mathrm{N}^{\mathrm{o}} \mathrm{de} \\
\text { materias }\end{array}$ & $\begin{array}{c}\text { Créditos } \\
\text { en el } \\
\text { módulo }\end{array}$ & $\begin{array}{c}\mathrm{N}^{\mathrm{o}} \mathrm{de} \\
\text { materias }\end{array}$ & $\begin{array}{c}\text { Créditos } \\
\text { en el } \\
\text { módulo }\end{array}$ \\
\hline Navarra & 9 & 18 & 9 & 25 & 2 & 21 \\
\hline Oviedo & 3 & 15 & 3 & 21 & 2 & 24 \\
\hline $\begin{array}{c}\text { País Vasco, UPV/ } \\
\text { EHU }\end{array}$ & 6 & 18 & 8 & 24 & 2 & 18 \\
\hline $\begin{array}{l}\text { Pontificia de } \\
\text { Comillas }\end{array}$ & 3 & 18 & 3 & 26 & 1 & 16 \\
\hline $\begin{array}{l}\text { Pontificia de } \\
\text { Salamanca }\end{array}$ & 8 & 32 & 2 & 8 & 2 & 20 \\
\hline $\begin{array}{c}\text { Pública de } \\
\text { Navarra (UPNA) }\end{array}$ & 3 & 14 & 5 & 30 & 3 & 16 \\
\hline Ramón Llull & 3 & 15 & 5 & 25 & 2 & 20 \\
\hline Salamanca & 3 & 15 & 3 & 27 & 2 & 18 \\
\hline $\begin{array}{l}\text { Santiago de } \\
\text { Compostela }\end{array}$ & 5 & 16 & $6-8$ & 26 & 2 & 18 \\
\hline UNED & 3 & 15 & 6 & 27 & 2 & 18 \\
\hline Valencia & 3 & 16 & 2 & 28 & 2 & 16 \\
\hline Valladolid & 3 & 12 & $7-9$ & 32 & 2 & 16 \\
\hline Zaragoza & 4 & 18 & 4 & 26 & 3 & 16 \\
\hline
\end{tabular}

Fuente: Elaboración propia

El número de créditos es muy parecido en casi todas (resulta lógico para ajustarse a las directrices), en el número de materias hay una mayor dispersión. En la Figura 3 se recoge el número de créditos en cada Módulo en cada universidad.

Puede comprobarse que el Módulo Específico es el que tiene mayor número de créditos y que el Módulo Genérico es el que menos. Esta tendencia es lógica teniendo en cuenta que el título está definido atendiendo a un modelo consecutivo, tal como se recoge en la Orden ECI 3858/2007 y que las Universidades cuentan con 8 créditos para añadir al módulo o módulos que determinen. Sin embargo, existen Universidades que han llegado a hacer pública una estructura que no es acorde a la señalada en la referida normativa. 


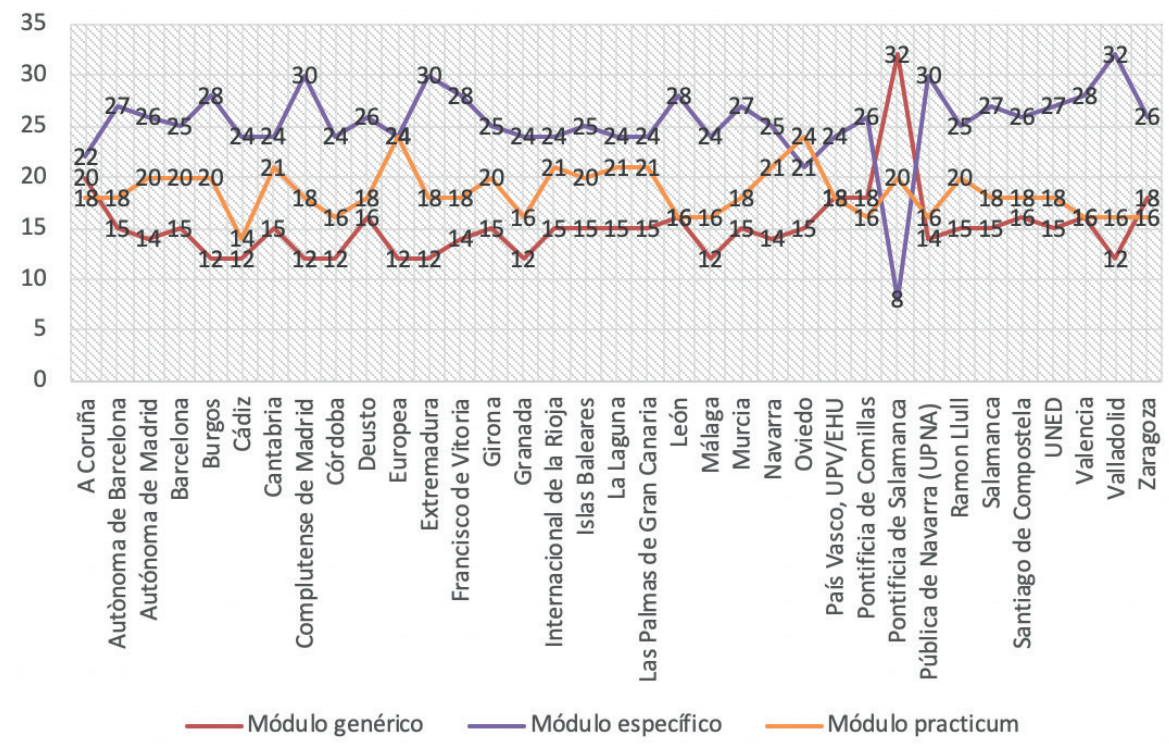

Figura 3. Créditos por módulos. Fuente: Elaboración propia.

\section{Objetivo específico 3. Describir la situación actual del profesorado del MUFPES}

En relación al perfil profesional del profesorado que imparte docencia en el MUFPES, se recoge en la Figura 4.

La mayoría (casi el $80 \%$ ), es profesorado universitario y el resto es de secundaria. Son destacables los 250 profesores de enseñanza secundaria que imparten docencia en el Máster de la Universidad de Granada, los 90 de la Universidad de Murcia o los 70 de la Universidad Complutense de Madrid. Por otro lado, nos encontramos con universidades donde el título está impartido en su totalidad por profesorado universitario como la Universidad Pontificia de Comillas o la Pública de Navarra, entre otras.

El dato recogido en esta gráfica está acorde con lo ya expresado por ANECA (2015, p. 32):

En referencia a las universidades públicas, la asignación de docencia es habitual que se haga por elección del profesorado interesado y siguiendo una jerarquía de antigüedad y categoría. 


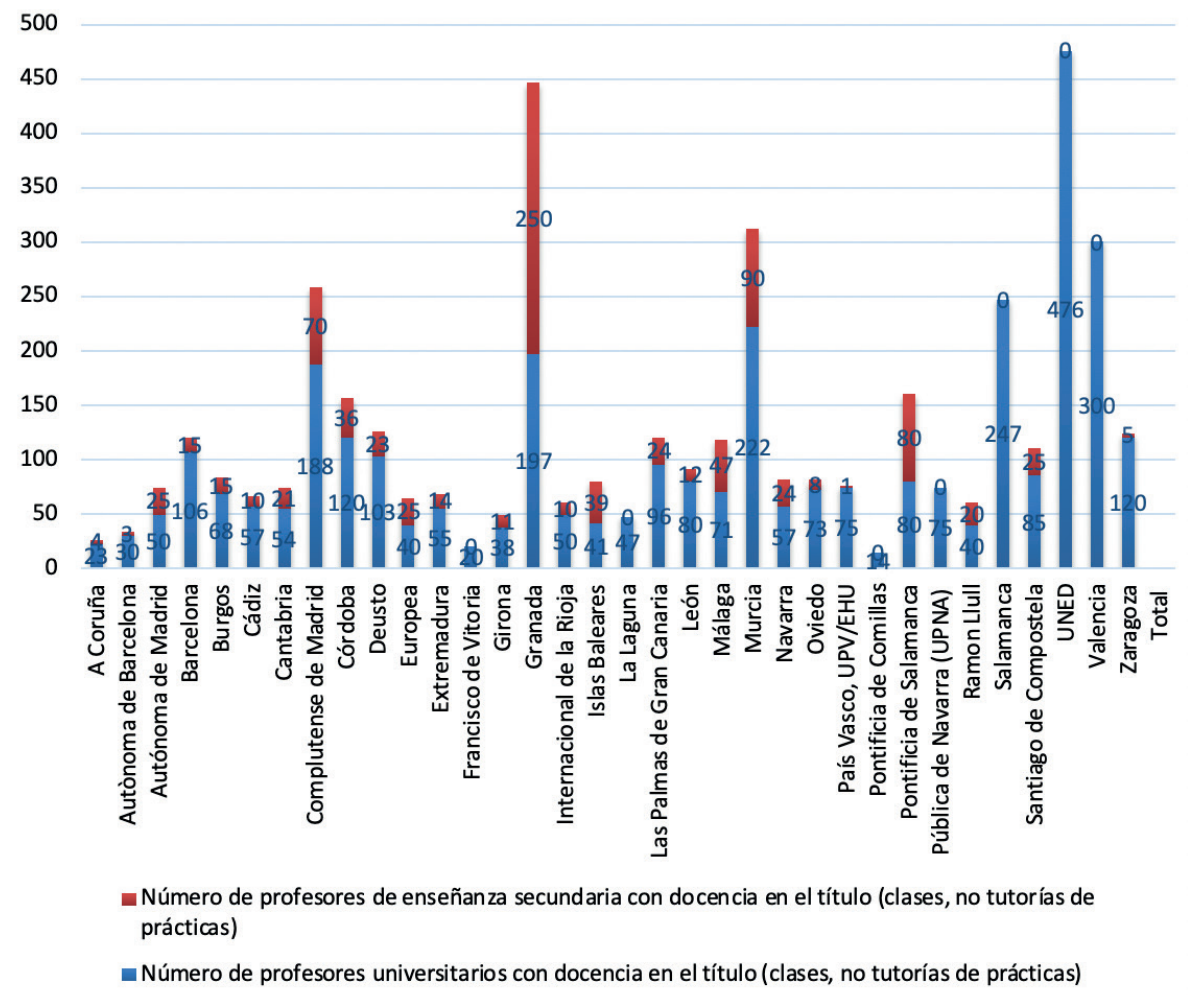

Figura 4. Profesores universitarios y de enseñanza secundaria. Fuente: Elaboración propia.

También es importante destacar otro dato extraído del informe en el que se indica lo siguiente (ANECA. 2015, p. 33):

En las universidades públicas parece existir un acuerdo tácito por el cual el módulo específico lo imparte profesorado del área disciplinar correspondiente, quien se encarga de la materia de Complementos para la formación disciplinar, en tanto que el profesorado de didácticas específicas imparte la materia de aprendizaje y enseñanza de la especialidad.

Este dato se apoya claramente en la información aportada por las Universidades y resulta cuestionado por algunos centros universitarios responsables de la formación inicial de profesorado. 


\section{Objetivo específico 4. Describir la situación de las prácticas y del Trabajo de Fin de Máster del MUFPES}

En relación a las prácticas, las respuestas se recogen en la Figura 5. 20

Figura 5. Créditos de la asignatura prácticas. Fuente: Elaboración propia.

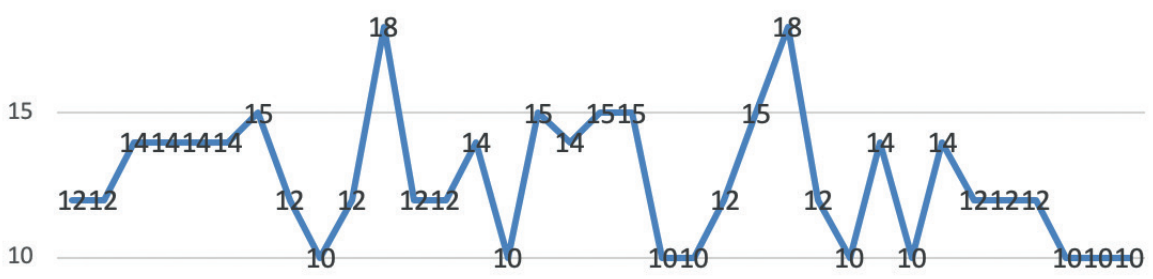

5

0

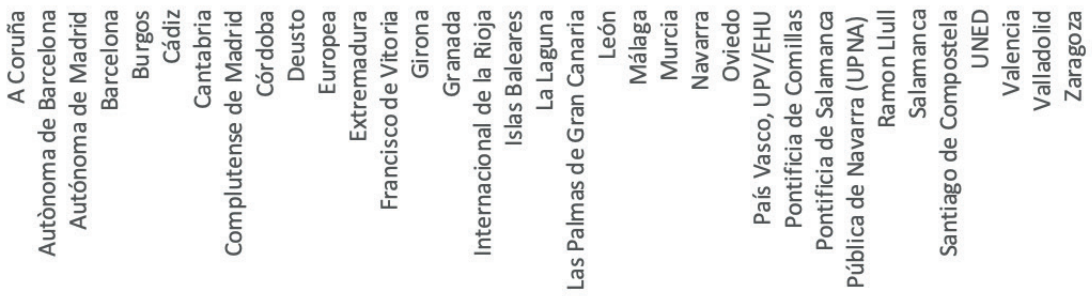

Podemos comprobar los créditos específicos que las diferentes universidades dedican a las prácticas, destacando los 18 de la Universidad Europea y de la Universidad de Oviedo.

El Practicum presenta una gran diversidad de tipologías en las diferentes universidades. Tras una exhaustiva revisión de las respuestas, podríamos identificar tres tipos:

- Una fase de vida en el centro de unas 150 horas de duración.

- Dos fases, entre tres y cinco semanas, o entre tres y seis semanas que pueden tener la siguiente estructura:

- Observación + Intervención

- Taller de habilidades docentes + Intervención

- Tres fases que suelen durar una semana. Cada fase tendría la estructura: 
- Observación + Intervención acompañada + Intervención autónoma

- Inmersión, planificación y observación + Intervención + Reflexión-Síntesis

En el Informe de ANECA (2015) se reconocían dos grandes modelos: en un solo periodo o en dos, por lo tanto, parece existir una evolución en este aspecto ya que aparecen tres.

La práctica totalidad de las Universidades tienen establecidos convenios de colaboración educativa para la realización de las prácticas docentes con las respectivas Comunidades Autónomas. Las convocatorias suelen ser anuales, o en algunos casos bienales, trienales y cuatrienales. Por lo general, las universidades no acreditan a los centros como centros de prácticas. En el 90\% de los casos, los centros concertados de enseñanza secundaria participan como centros de prácticas.

En relación con los requisitos para ser tutores de prácticas, existen muchas diferencias entre los distintos centros. Las clasificamos en las siguientes opciones:

- Profesores innovadores que practican metodologías acordes con los principios de la didáctica que se explican en las clases de la universidad.

- Ser profesor/a de plantilla.

- Acreditados por la Consejería de Educación de la Comunidad Autónoma.

- El requisito es la aceptación (con entusiasmo) por parte del tutor.

- Idoneidad docente.

- Dos o tres años de experiencia docente para los tutores.

- Pertenecer a algún cuerpo de la función docente o ser profesor interino y tener un mínimo de 5 años de experiencia docente.

- Pertenecer a la especialidad que oferta el Centro.

- Tener destino definitivo en el centro de prácticas.

- Al menos, con tres años de experiencia. Además, la dirección del Máster y la Comisión Académica utilizan algunos criterios más para seleccionar a los tutores.

En cuanto a la remuneración de la labor de tutoría en los centros de destino, encontramos algunas diferencias. En general, casi todas las universidades empezaron en el año 2010 a remunerar de alguna forma esta labor, 
pero, a partir de 2012, prácticamente dejó de hacerse en todas las Comunidades Autónomas. Hoy en día encontramos las siguientes opciones:

- No existe remuneración (son la mayoría).

- $100 €$.

- $170 €$ para los tutores y $255 €$ para los coordinadores.

- Se desvía el dinero de matrícula del estudiante al tutor de prácticas y al centro.

- La remuneración es por cada alumno/a tutelado/a, el pago de los 10 créditos de las prácticas.

- No existe remuneración, aunque sí se les remunera por el diseño e impartición de seminarios docentes a sus tutelados (100 euros).

Por otro lado, además o en lugar de la remuneración, las universidades ofrecen unos beneficios asociados a la participación de la tutela, en la mayoría de los casos son el acceso a servicios universitarios (carnet universitario, acceso a instalaciones, etc.), pero también se ofrecen los siguientes beneficios:

- Reconocimiento como mérito en los concursos y promociones profesionales.

- Participación gratuita en cursos de formación.

- Descuentos en cursos de la Universidad.

- Acreditación de horas impartidas.

- Certificación a tutores, invitación a ponencias, participación en los tribunales de TFM, etc.

El TFM es una asignatura de 6 créditos en todas las universidades. Las diferencias principales se encuentran en el tipo de trabajo que se solicita. Las respuestas analizadas las hemos clasificado en las siguientes opciones:

- Planificación curricular, Unidad Didáctica, Investigación educativa, Acción en temáticas transversales, Proyecto integrado o actividad complementaria. Innovación educativa con elaboración de materiales didácticos, Proyecto-proceso autográficos.

- Investigación o estudio con datos empíricos, procedentes de la experimentación de la unidad didáctica impartida.

- Investigación/innovación/síntesis reflexiva. 
- Programación didáctica que incorpore elementos de innovación o singularidad y se justifique teóricamente.

- Tres modalidades: revisión bibliográfica crítica, trabajo empírico o innovación y diseño y evaluación de un programa.

Los tipos de defensa de los trabajos son iguales en todas las universidades. Se trata de una defensa pública expositiva. La diferencia principal la marcan las universidades virtuales que utilizan sistemas de videoconferencia para suplir la presencialidad. En cualquier caso, se defiende ante un tribunal o comisión evaluadora.

\section{Conclusiones}

La primera y principal conclusión que se desprende del estudio realizado es la significativa heterogeneidad que puede encontrarse en la gestión y organización, estructura, perfil del profesorado y desarrollo del Practicum y del Trabajo de Fin de Máster. Del estudio actual no se desprende específicamente si esta variedad supone un factor positivo en cuanto a una formación más contextualizada y de mayor calidad para los egresados, o si, por el contrario, esta situación afecta negativamente al perfil de salida del estudiante que completa los estudios del Máster. En cualquier caso, parece oportuno realizar una revisión más profunda, por si las diferencias encontradas pueden conllevar una alteración de la filosofía con la que este título fue concebido para la formación inicial del profesorado de enseñanza secundaria.

Se observan diferencias en el número de especialidades que se ofertan en las distintas universidades. En este aspecto pueden incidir variables muy diversas: la adecuación de la oferta a las necesidades formativas de los docentes del entorno, la capacidad del profesorado y departamentos de cada universidad para afrontar esta tarea, el interés de la universidad por este tipo de estudios... No obstante, Ilamamos la atención sobre el hecho de que estos factores no nos permitan establecer un perfil homogéneo de los egresados.

En cuanto al número de plazas ofertadas y de estudiantes egresados desde que se implantó el título, parece necesario revisar con meticulosidad la necesidad real de profesorado de enseñanza secundaria. Se deben planificar las plantillas de las Comunidades Autónomas y preveer 
una oferta coherente con datos demográficos y de crecimiento poblacional, todo ello encaminado, como recogía el informe de ANECA (2015), a poder ofrecer una ratio de 25-30 alumnos por grupo, cuestión que, en la actualidad, es prácticamente imposible, a la luz de los datos obtenidos en el estudio.

También se desprende del estudio la necesidad de determinar un modelo formativo que dé respuesta a las necesidades que tiene un profesor de enseñanza secundaria a lo largo de su vida: en la formación inicial, en el acceso a la profesión docente, en la formación del profesorado en ejercicio...

En cuanto a la primera etapa del desarrollo profesional, el desarrollo del título en cada universidad ha atendido, en muchos casos, más a las necesidades de los Departamentos universitarios, que a las que serían prioritarias para los futuros profesores de enseñanza secundaria. Incluso, se han desvirtuado los planteamientos iniciales que, desde la propia Unión Europea, se proponían para actualizar esta formación y hacerlo acorde con la realidad social y educativa actual. Aunque la Orden ECI establece claramente la estructura por módulos del Máster, las universidades han adaptado esta estructura atendiendo a factores que, en muchos casos, poco, o nada, tienen que ver con las necesidades reales de formación y que atañen a otros factores relacionados con necesidades estructurales de las Universidades. De esta forma, se percibe una gran disparidad en la estructuración de los módulos, sobre todo en lo referido al número de materias de los mismos.

Podemos concluir que el estudio que se presenta en este artículo traza una clara línea de trabajo para la mejora de algunos aspectos del MUFPES, estableciendo claves concretas sobre su desarrollo que merecen un análisis más pormenorizado y que deben obligar, tanto a los responsables universitarios como a las autoridades educativas, a una mayor colaboración y participación en los planteamientos y decisiones que haya que abordar para la mejora del título en el conjunto de las universidades españolas, sin que se generen diferencias significativas a causa de la variedad de criterios que pueden plantearse por parte de las agencias evaluadoras para la acreditación y evaluación del mismo.

Para finalizar, queríamos proponer una serie de acciones futuras que han surgido de la investigación-reflexión realizada del MUFPES en el sistema universitario español:

- Reivindicar que la gestión y organización académica del MUFPES 
debe ser responsabilidad de los centros universitarios (facultades y escuelas) responsables de la formación inicial del profesorado.

- Recalcar, ante las autoridades educativas y agencias de evaluación el carácter profesionalizante del máster, que debería contar por ello con los mejores recursos y medios, tanto materiales como personales, por parte de las universidades, para una formación digna y de auténtica calidad.

- Reclamar a las autoridades educativas y universitarias, sobre todo a las agencias de evaluación, una clarificación precisa de los criterios de acreditación del título, así como una clara homogeneización de los mismos para todas las universidades y comunidades autónomas.

- Determinar un modelo formativo que dé respuesta a lo que entendemos por un profesor de secundaria.

- Establecer procesos definidos de formación, acreditación y reconocimiento de los tutores y centros de prácticas.

\section{Referencias}

ANECA (2015). Análisis de situación y propuestas de mejora. Sobre la implantación del Máster Universitario de Formación del Profesorado de Educación Secundaria Obligatoria, Bachillerato, Formación Profesional y Enseñanza de Idiomas. Recuperado de: http://www.didactica-ciencias-sociales.org/posgrado_fitxers/Informe_Master_Secundaria-ANECA.pdf

Blázquez, F. (Coord.) (2001). Sociedad de la información y educación. Mérida: Junta de Extremadura.

Consejo de la Unión Europea (2000). Consejo Europeo de Lisboa del 23 y 24 de marzo. Lisboa: Unión Europea.

Comisión Europea (2002). La Declaración de Copenhague. Declaración de los ministros europeos de formación y enseñanza profesional y la comisión europea sobre una mejor cooperación europea en materia de formación y enseñanza profesional. Copenhague: Comisión Europea.

Domingo, A. (2008). La práctica reflexiva en la formación inicial de maestros/as. Evaluación de un modelo. Tesis doctoral: Universitat Internacional de Catalunya.

Eurydice (2009). Key data on Education in Europe 2009. Brussels: Directorate-General of Education and Culture.

Guttman, C. (2003). Education in and for the Information Society. Paris: UNESCO.

Hargreaves, A. (2003). Teaching in the Knowledge Society. Education in the Age of Insecurity. New York: Teacher College Press.

Ley Orgánica 2/2006, de 3 de mayo, de Educación. Boletín Oficial del Estado, 106, de 4 de mayo, pp. 17158-17207. 
MEC (2015). Datos y Cifras del sistema universitario español. Curso 2014/2015. Madrid: Solana e hijos, A.G., S.A.U.

Marina, J.A, Pellicer, C. y Manso, J. (2015). Libro blanco de la profesión docente y su entorno escolar. Ministerio de Educación, Cultura y Deporte. Recuperado de: http:// www.mecd.gob.es/mecd/dms/mecd/destacados/libro-blanco/libro-blanco-profesiondocente.pdf

Martínez, Z. y Villardón-Gallego, L. (2015). La imagen del profesor de Educación Secundaria en la formación inicial. Revista de currículum y formación del profesorado, 19(1), 452-467.

Murillo, F. J. (2006). Modelo Innovadores en la Formación Inicial Docente. Santiago de Chile: UNESCO.

Orden ECl/3858/2007, de 27 de diciembre, por la que se establecen los requisitos para la verificación de los títulos universitarios oficiales que habiliten para el ejercicio de las profesiones de Profesor de Educación Secundaria Obligatoria y Bachillerato, Formación Profesional y Enseñanzas de Idiomas.

Real Decreto 1393/2007, de 29 de octubre, por el que se establece la ordenación de las enseñanzas universitarias oficiales.

Real Decreto 1834/2008, de 8 de noviembre, por el que se definen las condiciones de formación para el ejercicio de la docencia en la educación secundaria obligatoria, el bachillerato, la formación profesional y las enseñanzas de régimen especial y se establecen las especialidades de los cuerpos docentes de enseñanza secundaria.

Real Decreto 665/2015, de 17 de julio, por el que se desarrollan determinadas disposiciones relativas al ejercicio de la docencia en la Educación Secundaria Obligatoria, el Bachillerato, la Formación Profesional y las enseñanzas de régimen especial, a la formación inicial del profesorado y a las especialidades de los cuerpos docentes de Enseñanza Secundaria.

Tiana, A. (2013). Los cambios recientes en la formación inicial del profesorado en España: una reforma incompleta. Revista Española de Educación Comparada, 22, 39-58

Valle, J. M., \& Manso, J. (2011). La nueva formación inicial del profesorado de Educación Secundaria: modelo para la selección de buenos centros de prácticas. Revista de Educación, 354, 267-290. 
